\title{
Écart de mobilité : estimation des seuils de mobilité requis pour maîtriser le SRAS-CoV-2 au Canada
}

\author{
Kevin A. Brown PhD, Jean-Paul R. Soucy MSc, Sarah A. Buchan PhD, Shelby L. Sturrock MSc, Isha Berry MSc, \\ Nathan M. Stall MD, Peter Jüni MD, Amir Ghasemi PhD, Nicholas Gibb MSc, Derek R. MacFadden MD, \\ Nick Daneman MD
}

Citation : CMAJ 2021 April 26;193:E592-600. doi : 10.1503/cmaj.210132-f; diffusion hâtive le 7 avril 2021

Voir la version anglaise de l'article ici : www.cmaj.ca/lookup/doi/10.1503/cmaj.210132

\section{RÉSUMÉ}

CONTEXTE : Les interventions non pharmacologiques demeurent le principal moyen de maîtriser le coronavirus du syndrome respiratoire aigu sévère 2 (SRAS-CoV-2) d'ici à ce que la couverture vaccinale soit suffisante pour donner lieu à une immunité collective. Nous avons utilisé des données de mobilité anonymisées de téléphones intelligents afin de quantifier le niveau de mobilité requis pour maîtriser le SRAS-CoV-2 (c.à-d., seuil de mobilité), et la différence par rapport au niveau de mobilité observé (c.-à-d., écart de mobilité).

MÉTHODES : Nous avons procédé à une analyse de séries chronologiques sur l'incidence hebdomadaire du SRASCoV-2 au Canada entre le 15 mars 2020 et le 6 mars 2021. Le paramètre mesuré était le taux de croissance hebdoma- daire, défini comme le rapport entre les cas d'une semaine donnée et ceux de la semaine précédente. Nous avons mesuré les effets du temps moyen passé hors domicile au cours des 3 semaines précédentes à l'aide d'un modèle de régression log-normal, en tenant compte de la province, de la semaine et de la température moyenne. Nous avons calculé le seuil de mobilité et l'écart de mobilité pour le SRAS-CoV-2.

RÉSULTATS : Au cours des 51 semaines de l'étude, en tout, 888751 personnes ont contracté le SRAS-CoV-2. Chaque augmentation de $10 \%$ de l'écart de mobilité a été associée à une augmentation de $25 \%$ du taux de croissance des cas hebdomadaires de SRAS-CoV-2 (rapport 1,25 , intervalle de confiance à $95 \%$ 1,20-1,29). Comparativement à la mobilité prépandémique de référence de $100 \%$, le seuil de mobilité a été plus élevé au cours de l'été (69\%, écart interquartile [EI] 67\%-70\%), et a chuté à $54 \%$ pendant l'hiver 2021 (EI 52\%$55 \%)$; un écart de mobilité a été observé au Canada entre juillet 2020 et la dernière semaine de décembre 2020.

INTERPRÉTATION : La mobilité permet de prédire avec fiabilité et constance la croissance des cas hebdomadaires et il faut maintenir des niveaux faibles de mobilité pour maîtriser le SRAS-CoV-2 jusqu'à la fin du printemps 2021. Les données de mobilité anonymisées des téléphones intelligents peuvent servir à guider le relâchement ou le resserrement des mesures de distanciation physique provinciales et régionales.
$\mathbf{L}$

e bilan mondial de la maladie à coronavirus 2019 (COVID19) continue de s'alourdir, malgré la promesse que recèlent les vaccins récemment approuvés. Une recrudescence s'observe dans de nombreux pays de l'hémisphère nord, y compris au Canada, ce qui pourrait alourdir considérablement le fardeau avant que la vaccination soit suffisante pour donner lieu à une immunité collective. Les interventions non pharmacologiques de la santé publique, y compris la distanciation physique, demeurent le principal moyen de maîtriser la COVID-19 à l'échelle des populations ${ }^{1}$. Depuis le début de la deuxième vague, qui a commencé en septembre 2020, des enquêtes ont laissé entendre qu'une majorité de personnes au Canada appuie et respecte les restrictions imposées par les gouvernements ${ }^{2}$, et qu'elles sont nombreuses à approuver un resserrement des mesures pour maîtriser la transmission communautaire du coronavirus du syndrome respiratoire aigu sévère (SRAS-CoV-2), l'agent viral à l'origine de la COVID-193.

Le SRAS-CoV-2 se propage principalement lors de contacts étroits avec des personnes porteuses ${ }^{4}$. Comme avec toute maladie infectieuse, le taux de contacts est le principal moteur de la transmission 
du SRAS-CoV-25 ${ }^{5}$ Les données de mobilité qui rendent compte de l'activité humaine par un suivi anonymisé des téléphones intelligents pourraient raisonnablement rendre compte des taux de contacts hors domicile; ces données peuvent fournir des renseignements fiables sur les taux de contacts plus rapidement que les enquêtes sur l'emploi du temps et les recherches de contacts ${ }^{6-8}$.

Les données de mobilité agrégées des téléphones intelligents sont fournies par plusieurs développeurs de logiciels et ont servi à mesurer l'impact des politiques de mobilité au Canada ${ }^{9}$, l'efficacité des mesures de confinement visant à réduire la propagation du SRAS-CoV-2 $2^{10-12}$, et les lacunes des mesures trop locales $^{13}$. Les données de mobilité sont utiles pour mesurer l'effet du confinement sur les comportements, mais en soi, elles ne disent pas aux décideurs si les restrictions en place à un moment donné suffisent pour limiter la propagation du SRAS-CoV-2. Dans cette étude, nous avons évalué le lien entre les données de mobilité des téléphones intelligents et la propagation du SRASCoV-2 au Canada, à l'échelle du pays et des provinces, entre mars 2020 et mars 2021. Nous avons aussi voulu mesurer le niveau de mobilité requis pour maîtriser la COVID-19 (c.-à-d., seuil de mobilité) et la différence entre les niveaux de mobilité observés et le seuil de mobilité (c.-à-d., écart de mobilité). Selon notre hypothèse, des niveaux de mobilité plus bas pourraient être requis l'hiver dans les provinces où les villes sont plus populeuses, en comparaison avec les provinces plus rurales l'été14.

\section{Méthodes}

\section{Modèle d'étude}

Nous avons procédé à une analyse de séries chronologiques pour mesurer l'effet de la mobilité sur l'incidence hebdomadaire du SRAS-CoV-2 au Canada entre le 15 mars 2020 et le 6 mars 2021. L'étude a été réalisée à 2 niveaux, national et provincial. Pour les analyses concernant le niveau provincial, seules les semaines où l'incidence était de plus de 20 cas au cours de la semaine précédente ont été incluses, parce que les taux d'incidence des semaines où les nombres de cas étaient faibles risquaient d'être fortement affectés par le phénomène d'importation de cas et les éclosions sporadiques. Sur la base de l'inspection visuelle de l'ajustement du modèle, nous avons inclus uniquement les provinces ou les territoires pour lesquels au moins $50 \%$ des semaines pouvaient être incluses dans les analyses provinciales, pour nous assurer que les estimations spécifiques aux provinces étaient exactes. Lorsque plusieurs segments disjoints de semaines admissibles d'une même province pouvaient être inclus dans l'analyse, nous n'avons inclus que le segment le plus long.

\section{Paramètres}

Nous avons établi le nombre de cas et la positivité des tests de SRAS-CoV-2 hebdomadaires dans chaque province à partir des données du COVID-19 Canada Open Data Working Group ${ }^{15}$ pour les 51 semaines de la période allant du 15 mars 2020 au 6 mars 2021. Les paramètres ont été agrégés par semaine pour tenir compte des modes de présentation quotidiens illustrés par les données de signalement des cas au Canada ${ }^{16}$. L'Open Data Working Group obtient et compile les nombres de cas quotidiens signalés partout au pays par les agences de santé publique provinciales, les médias accrédités et les comptes officiels des médias sociaux. Les semaines commençaient le dimanche et se terminaient le samedi.

Selon notre hypothèse, la mobilité allait avoir un effet sur la dynamique du SRAS-CoV-2 en ce qui concerne les changements de taux, plutôt que de taux absolus d'infections, notre paramètre était donc le taux de croissance hebdomadaire, représenté par le rapport entre les cas de SRAS-CoV-2 au cours d'une semaine donnée et ceux de la semaine précédente. Un taux de croissance hebdomadaire égal à 1 signifie que l'incidence était stable par rapport à la semaine précédente, et un taux de croissance hebdomadaire supérieur à 1 signifie une hausse du nombre de cas. Comme les données de surveillance sont sujettes à un phénomène de sous-détection instationnaire, nous avons conçu un taux de croissance corrigé, égal au taux de croissance des cas hebdomadaires multiplié par le taux de croissance hebdomadaire de la positivité des tests.

\section{Données de mobilité}

Les données de mobilité provinciales des téléphones intelligents proviennent des rapports de Google en libre accès sur la mobilité de la communauté ${ }^{17}$ mis à jour quotidiennement. Ces données sont recueillies auprès d'utilisateurs de Google Maps qui ont activé leur historique de localisation, une fonction qui est désactivée par défaut. La principale exposition visée était le temps moyen passé hors domicile au cours des 3 semaines précédentes, un paramètre validé qui est un solide indicateur de l'instauration et de la levée des interventions non pharmacologiques de la santé publique ${ }^{18}$. Ce temps de décalage a été choisi sur la base d'une période tampon de 10 jours entourant le pic de corrélation connu entre la mobilité et le taux de croissance des cas au $11^{\mathrm{e}}$ jour ${ }^{12}$.

Nous avons défini le niveau de référence des données de mobilité comme la valeur médiane pour la période de 5 semaines allant du 3 janvier au 6 février 2020, soit le mois précédant la confirmation du premier cas de transmission communautaire au Canada (le 5 mars, en Colombie-Britannique) et avant la première fermeture d'école au Canada (le 15 mars en Ontario). Nous avons fait une remise à l'échelle des données de mobilité résidentielles de Google (formule : $100 \times[1-X / 30]$, estimant que l'hiver, la population canadienne passe $30 \%$ de son temps hors domicile), de sorte que les niveaux de la période de référence représentaient $100 \%$, avec un écart allant de $0 \%$ (aucune mobilité hors domicile) à plus de $100 \%{ }^{8,9}$. Pour tracer le graphique de la mobilité hors domicile, nous avons lissé les valeurs d'indices à l'aide d'une spline pénalisée avec un nœud pour chaque période de 2 semaines $^{19}$, et nous les avons superposées à une moyenne mobile de 7 jours.

\section{Covariables}

En plus de la mobilité, nous avons tenu compte de la semaine et de la température moyenne (en degrés Celsius) au cours d'une période de décalage de 3 semaines pour les villes les plus populeuses de chaque province, selon les données d'Environnement Canada ${ }^{20}$. À des fins descriptives, nous avons regroupé les semaines d'un même trimestre : mars 2020, avril-juin 2020, juillet-septembre 2020, octobre-décembre 2020 et janviermars 2021. 


\section{Analyse statistique}

Nous avons décrit les taux de croissance des cas, la positivité et les niveaux de mobilité non résidentiels hebdomadaires pour les provinces et les trimestres, avec médiane et écart interquartile (EI). Nous avons modélisé le logarithme de la croissance hebdomadaire des cas de SRAS-CoV-2 à l'aide d'un modèle de régression gaussien. Les coefficients de corrélation de ce modèle ont été potentialisés et représentent les rapports des taux de croissance (RTC). Les variables pour lesquelles les RTC étaient supérieurs à 1 étaient associées à une croissance accélérée. Celles pour lesquelles les RTC étaient inférieurs à 1 étaient associées à un ralentissement de la croissance. Pour les paramètres primaires (non corrigés en fonction de la positivité des tests) et secondaires (corrigés en fonction de la positivité des tests), nous avons conçu 2 modèles de régression : un modèle non ajusté qui incluait la mobilité hors domicile des 3 semaines précédentes et une spline pénalisée pour la semaine (avec un nœud pour chaque période de 2 mois) et un modèle ajusté qui tenait compte aussi de la température moyenne des 3 semaines précédentes comme covariable linéaire. Tous les modèles ont été ajustés à l'aide de la trousse mgcv en $\mathrm{R}$ (détails sur le modèle à l'annexe 1 , accessible en anglais au www.cmaj.ca/lookup/doi/10.1503/ cmaj.210132/tab-related-content) ${ }^{19,21}$.

À partir du modèle ajusté pour le lien entre le taux de croissance des cas de SRAS-CoV-2 et la mobilité, nous avons estimé le seuil de mobilité à partir duquel la croissance des cas de SRASCoV-2 cesserait. Le calcul du seuil de mobilité est décrit à l'annexe 1. Nous avons défini l'écart de mobilité comme la différence entre la mobilité observée et le seuil de mobilité. L'écart de mobilité peut être interprété comme la réduction incrémentielle estimée de la mobilité qui aurait été requise pour maîtriser le taux de croissance de cas de SRAS-CoV-2 dans une province donnée au cours d'une semaine donnée.

À des fins de comparaison entre les modèles, nous avons mesuré plusieurs paramètres des tests diagnostiques des modèles, y compris l'autocorrélation résiduelle de Pearson à 1 semaine de décalage, le modèle $R^{2}$ ( 1 - écart modèle $_{\text {écart }}$ nul), les degrés de liberté estimés (en tenant compte des pénalités) et le critère d'information d'Akaike (AIC) (égal à la somme de l'écart et des degrés de liberté), une mesure de l'ajustement des modèles où les valeurs moindres indiquent plus de parcimonie et un meilleur ajustement des modèles.

\section{Approbation éthique}

Toutes les données utilisées dans cette étude étant du domaine public, il n'a pas été nécessaire de la soumettre à l'examen du comité d'éthique de la recherche de l'Université de Toronto.

\section{Résultats}

Pendant la période de 51 semaines (du 15 mars 2020 au 6 mars 2021), on a dénombré 888751 cas de SRAS-CoV-2 au Canada (tableau 1). Tous les cas ont été inclus dans l'analyse nationale et $881009(99,1 \%)$ cas ont été inclus dans les analyses

Tableau 1 : Positivité des tests de SRAS-CoV-2, taux de croissance des cas et mobilité hebdomadaires au Canada du 15 mars 2020 au 6 mars 2021

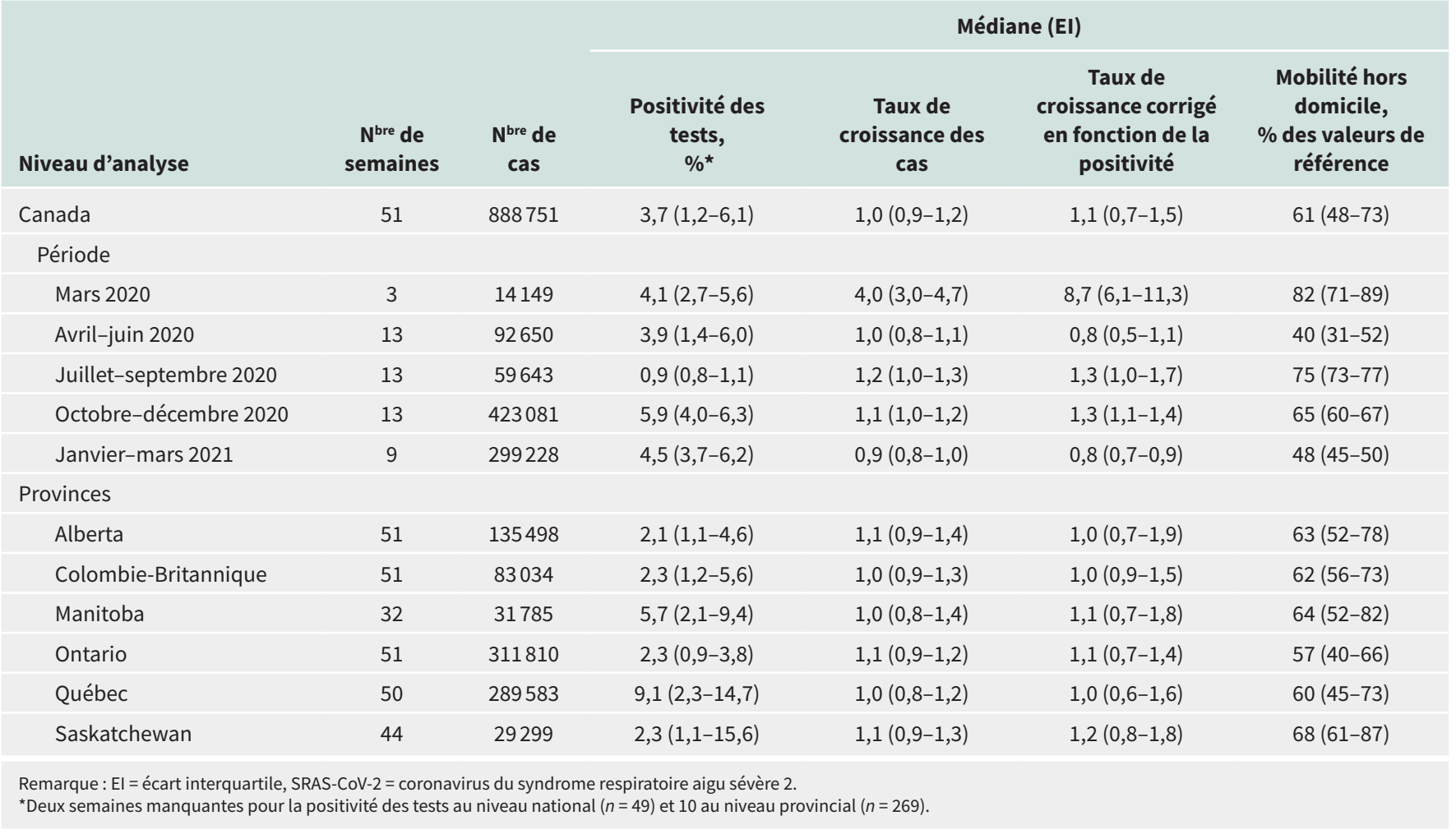


A

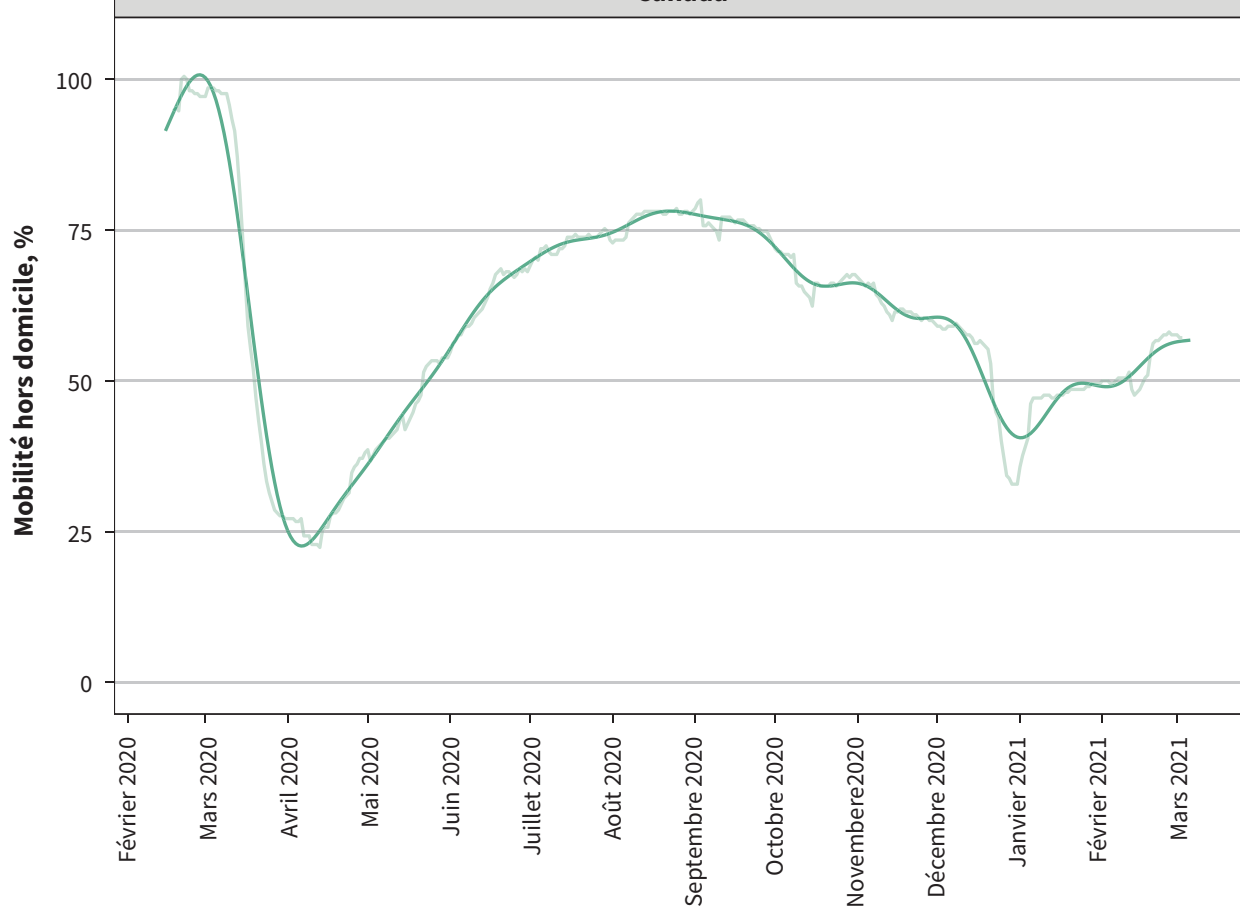

Date

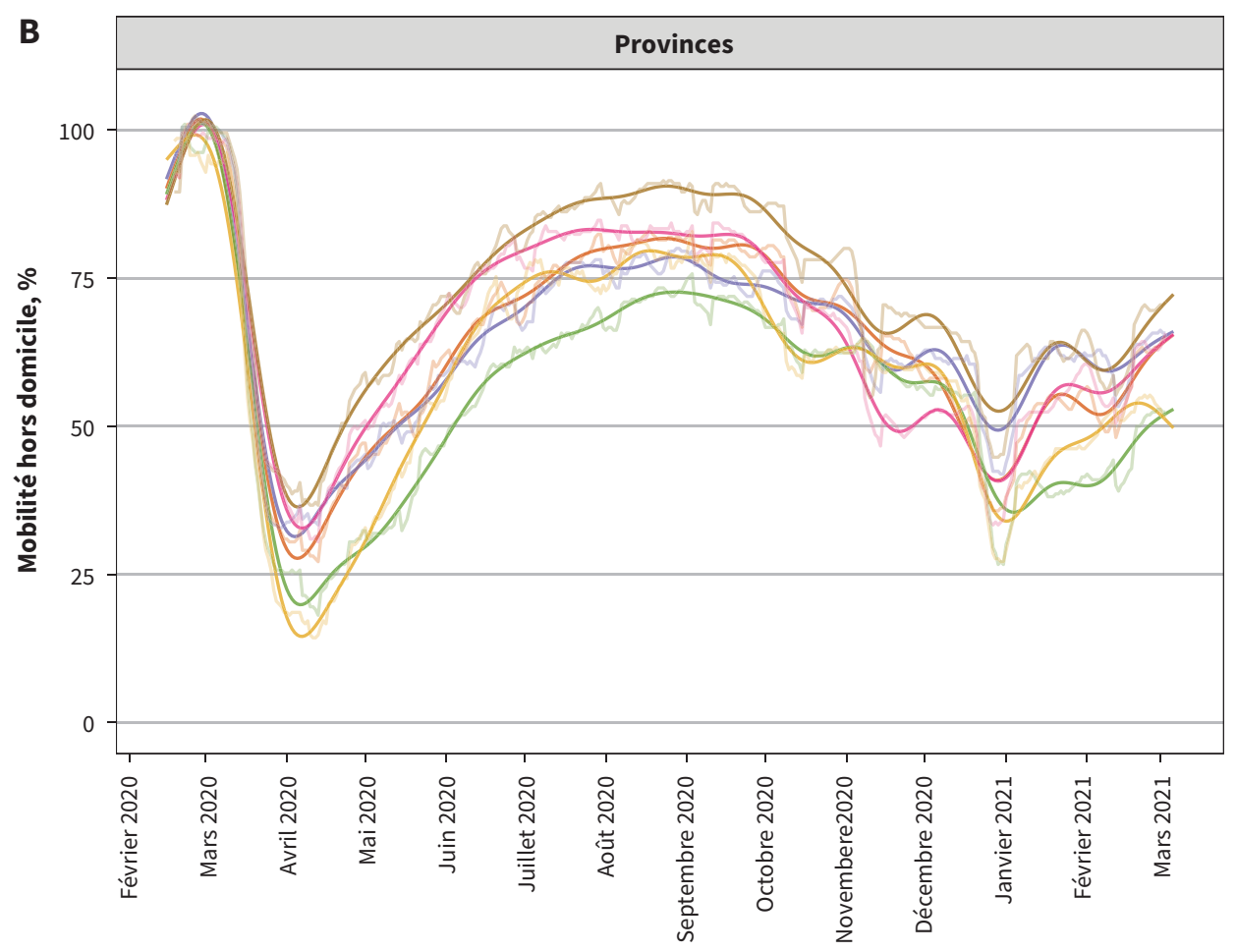

Date

Figure 1 : Mobilité hors domicile (A) au Canada et (B) dans 6 provinces canadiennes du 5 février 2020 au 6 mars 2021 . Remarque : L'indice de mobilité hors domicile est une mesure de la durée moyenne passée hors domicile selon les données de mobilité des téléphones intelligents (indice mis à l'échelle de façon à ce que les niveaux de la période de référence [du 3 janvier au 6 février 2020] représentent 100\%). Les valeurs indices sont lissées à l'aide d'une spline pénalisée, avec un nœud pour chaque période de 2 semaines (ligne en gras), et superposées à une moyenne mobile de 7 jours (ligne pâle). 
provinciales, qui totalisaient 279 semaines-provinces admissibles. L'Ontario ( $n=311810)$ et le Québec $(n=289583)$ ont eu les plus grands nombres de cas de SRAS-CoV-2 admissibles.

Partout au Canada, la mobilité hors domicile a chuté rapidement en mars 2020 pour atteindre un creux de $23 \%$ à la semaine du 5 avril (figure 1). La mobilité a augmenté au cours de l'été 2020 pour atteindre des niveaux s'approchant des valeurs de référence à la semaine du 23 août (78\%), puis a diminué lentement pendant les mois d'automne et rapidement en décembre 2020. Le Manitoba s'est démarqué avec des niveaux de mobilité plus bas que ceux des autres provinces durant l'automne 2020.

\section{Mobilité et taux de croissance des cas de SRAS-COV-2}

Dans le modèle national, après ajustement pour tenir compte de la date et des effets de la température, chaque augmentation de $10 \%$ de la mobilité a été associée à une augmentation de $25 \%$ du taux de croissance hebdomadaire (RTC ajusté 1,25 par $10 \%$ d'augmentation de la mobilité, intervalle de confiance [IC] à $95 \% 1,20-1,29$ ) (tableau 2 et figure 2 ). Les hausses de température moyenne hebdomadaire ont été significativement associées à une baisse des taux de croissance des cas de SRAS-CoV-2 (RTC 0,83 par augmentation de $5{ }^{\circ} \mathrm{C}, \mathrm{IC}$ à $95 \% 0,75-0,93)$. Les tests diagnostiques des modèles ont indiqué des taux faibles d'autocorrélation résiduelle (corrélation de Pearson, modèle ajusté $=0,15)$, et que l'ajustement des modèles était solide $\left(R^{2}\right.$, modèle ajusté $\left.=81,6 \%\right)$. Le paramètre corrigé en fonction de la positivité était manquant pour 2 semaines; il restait donc 49 semaines pour l'analyse. Les résultats ont été similaires avec ce paramètre (RTC ajusté 1,35 par $10 \%$ d'augmentation de la mobilité, IC à $95 \%$ $1,17-1,55)$, même si l'ajustement intégral du modèle était moins bon $\left(R^{2}=56,2 \%\right)$.

Pour les analyses provinciales, après ajustement en fonction de la date et des effets de la température, chaque augmentation de $10 \%$ de la mobilité a été associée à une augmentation de $20 \%$ du taux de croissance hebdomadaire (RTC ajusté 1,20 , IC à $95 \% 1,16-1,24)$ et la hausse de température a été associée à des taux de croissance moindres (RTC 0,88 par $5{ }^{\circ} \mathrm{C}$ de hausse de température, IC à $\left.95 \% 0,86-0,91\right)$.

Tableau 2 : Facteurs ayant influé sur les taux de croissance hebdomadaires des cas de SRAS-CoV-2 et les taux de croissance hebdomadaires corrigés en fonction de la positivité au Canada entre le 15 mars 2020 et le 6 mars 2021

\begin{tabular}{|c|c|c|c|c|}
\hline \multirow[b]{2}{*}{ Variable } & \multicolumn{2}{|c|}{$\begin{array}{l}\text { Niveau national* } \\
\quad n=51\end{array}$} & \multicolumn{2}{|c|}{$\begin{array}{l}\text { Niveau provincial* } \\
\quad n=279\end{array}$} \\
\hline & Non ajustée† & Ajustéeł & Non ajustée† & Ajustéeł \\
\hline \multicolumn{5}{|l|}{ Taux de croissance hebdomadaires } \\
\hline \multicolumn{5}{|l|}{ Coefficients, RTC (IC à 95\%) } \\
\hline $\begin{array}{l}\text { Mobilité hors domicile moyenne des } 3 \text { semaines } \\
\text { précédentes, par augmentation de } 10 \%\end{array}$ & $1,19(1,13-1,24)$ & $1,25(1,20-1,29)$ & $1,16(1,12-1,20)$ & $1,20(1,16-1,24)$ \\
\hline Température, par augmentation de $5^{\circ} \mathrm{C}$ & & $0,83(0,75-0,93)$ & & $0,88(0,86-0,91)$ \\
\hline \multicolumn{5}{|l|}{ Caractéristiques des modèles } \\
\hline Autocorrélation résiduelle & 0,14 & 0,15 & 0,20 & 0,09 \\
\hline Complexité des modèles (degrés de liberté) & 7,8 & 7,5 & 23,9 & 15,6 \\
\hline Qualité de l'ajustement, $R^{2}(\%)$ & 81,8 & 81,6 & 33,3 & 38,2 \\
\hline \multicolumn{5}{|l|}{ Coefficients, RTC (IC à 95\%) } \\
\hline $\begin{array}{l}\text { Mobilité hors domicile moyenne des } 3 \text { semaines } \\
\text { précédentes, par augmentation de } 10 \%\end{array}$ & $1,27(1,10-1,47)$ & $1,35(1,17-1,55)$ & $1,13(1,08-1,19)$ & $1,29(1,21-1,38)$ \\
\hline Température, par augmentation de $5^{\circ} \mathrm{C}$ & & $0,73(0,56-0,94)$ & & $0,89(0,84-0,94)$ \\
\hline \multicolumn{5}{|l|}{ Caractéristiques des modèles } \\
\hline Autocorrélation résiduelle & 0,04 & 0,03 & 0,15 & 0,10 \\
\hline Complexité des modèles (degrés de liberté) & 7,8 & 8,2 & 3,0 & 15,0 \\
\hline Qualité de l'ajustement, $R^{2}(\%)$ & 54,4 & 56,2 & 9,7 & 15,6 \\
\hline Critère d’ajustement des modèles (AIC) & 34,6 & 33,3 & 509,0 & 490,5 \\
\hline
\end{tabular}



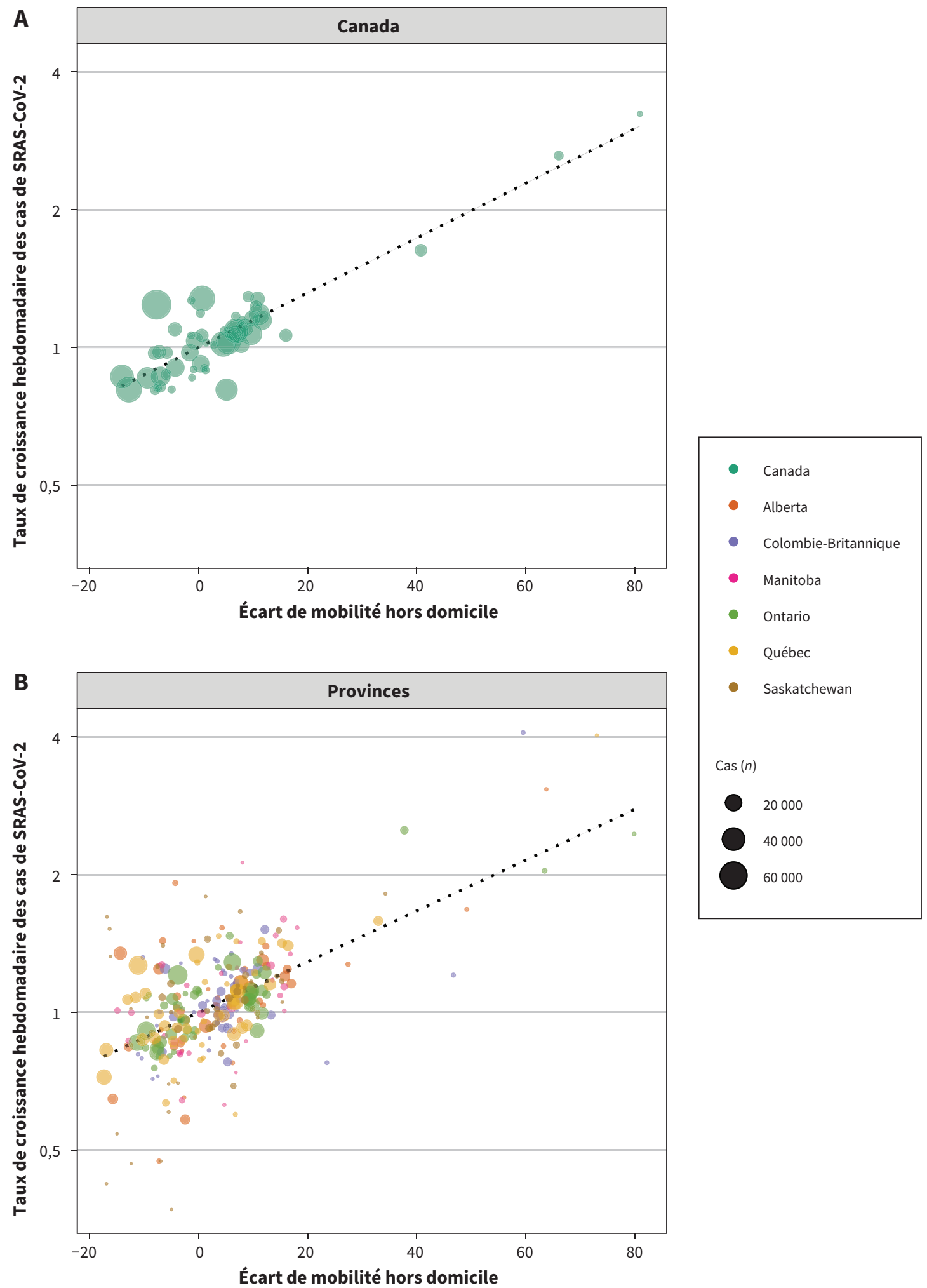

Figure 2 : Lien ajusté entre la mobilité hors domicile et le taux de croissance des cas de coronavirus du syndrome respiratoire aigu sévère 2 (SRASCoV-2) dans 6 provinces canadiennes entre le 15 mars 2020 et le 6 mars 2021. Le taux de croissance hebdomadaire des cas de SRAS-CoV-2 (nombre de cas d'une semaine donnée/semaine précédente) est étroitement lié à la mobilité hors domicile des 3 semaines précédentes. Dans l'analyse ajustée au niveau canadien, chaque augmentation de $10 \%$ de la mobilité hors domicile a été associée à une augmentation de $25 \%$ du taux de croissance (rapport des taux de croissance [RTC] 1,25, intervalle de confiance [IC] à $95 \% 1,20-1,29)$. Dans l'analyse provinciale ajustée, chaque augmentation de $10 \%$ de la mobilité a été associée à une augmentation de $20 \%$ du rapport des taux de croissance (RTC 1,20, IC à 95\% 1,16-1,24). Les associations sont représentées en pointillé. 
L'ajustement des modèles au niveau provincial a été plus faible $\left(R^{2}\right.$, modèle ajusté $\left.=38,2 \%\right)$. Le paramètre corrigé en fonction de la positivité était manquant pour 10 semaines, il restait donc 269 semaines-provinces pour l'analyse. Un lien solide entre la mobilité et le taux de croissance corrigé en fonction de la positivité a également été révélé (RTC ajusté 1,29 par 10\% d'augmentation de la mobilité, IC à $95 \%$ $1,21-1,38)$.

\section{Seuil de mobilité et écart de mobilité}

Nous avons utilisé les modèles ajustés au niveau national et provincial pour mesurer les seuils de mobilité (figure 3). Le seuil de mobilité national a varié nettement au cours de la pandémie pour atteindre un sommet durant l'été (médiane $71 \%$, El $69 \%-72 \%)$, et il a décliné tout au long de l'automne, jusqu'à $54 \%$ (EI 52\%-55\%) durant l'hiver. On a aussi observé des variations des seuils de mobilité provinciaux estimés; l'Ontario (50\%, El 46\%-59\%) et le Québec (54\%, El 52\%-63\%) ont eu les seuils les plus bas.

L'écart de mobilité dans les provinces canadiennes a connu différentes phases au cours de la pandémie (figure 3). Lors du début de la pandémie en mars 2020, la mobilité hors domicile excédait le seuil de mobilité. Les mesures de confinement strictes ont rapidement entraîné une baisse sous le seuil de mobilité et permis de maîtriser le taux de croissance des cas de SRAS-CoV-2 (avril-mai). Le relâchement des mesures de confinement à la fin du printemps a coïncidé avec une hausse des seuils de mobilité, mais la mobilité a rapidement augmenté jusqu'à excéder les seuils requis pour maîtriser le SRAS-CoV-2 à l'été 2020. Les seuils de mobilité ont diminué pendant l'automne et la mobilité est demeurée au-dessus du seuil, coïncidant avec une remontée du nombre de cas. En novembre 2020, le Manitoba a nettement réduit la mobilité à des niveaux inférieurs au seuil de mobilité, tandis que la mobilité au Canada n'a diminué sous le seuil qu'au cours de la dernière semaine de décembre.

\section{Interprétation}

Notre évaluation des prédicteurs des taux de croissance hebdomadaires des cas de SRAS-CoV-2 au Canada montre que les réductions de la mobilité sont en étroite corrélation avec la maîtrise des taux de croissance des cas de SRAS-CoV-2 des 3 semaines subséquentes et suggère que des réductions plus substantielles de la mobilité auraient été requises pour maîtriser la transmission du SRAS-CoV-2 au cours de l'automne 2020. Nous avons mis au point des méthodes de calcul du niveau de mobilité estimé requis pour maîtriser le SRAS-CoV-2 au Canada (seuil de mobilité) et de la réduction estimée de la mobilité requise pour maîtriser la croissance des cas de SRAS-CoV-2 (écart de mobilité).

Cette étude s'inspire de travaux qui ont révélé l'existence de liens étroits entre les mesures de distanciation physique et l'incidence du SRAS-CoV-2 ${ }^{11,22,23}$. Des études sur les données de mobilité des téléphones intelligents montrent que des changements de la mobilité permettent de prédire l'incidence précise du SRAS-CoV-2 des 1 à 3 semaines subséquentes ${ }^{12}$. Des données de mobilité plus détaillées suggèrent que les restaurants qui servent des repas sur place ou pour emporter (exposant probablement leurs travailleurs plus que leurs clients à un risque), les salles de sport et les cafés sont des vecteurs particulièrement importants dans l'incidence du SRAS-CoV-2 aux États-Unis ${ }^{24}$. Il est possible de mesurer le seuil de mobilité requis pour maîtriser la propagation du SRASCoV- $2^{25}$. Nous avons montré que les réductions de la mobilité requises varient selon les saisons : des réductions relativement modestes ont été nécessaires pour maîtriser le SRASCoV-2 durant l'été 2020, mais des réductions plus importantes sont requises depuis l'automne.

Comme pour plusieurs agents pathogènes respiratoires ${ }^{26,27}$, nous avons observé une variation saisonnière substantielle du risque d'infection au SRAS-CoV-2. On ne s'entend pas sur les facteurs qui en sous-tendent l'incidence accrue durant l'hiver. Entre autres hypothèses, mentionnons des facteurs liés aux comportements humains, particulièrement le fait de passer plus de temps à l'intérieur dans des endroits mal ventilés, l'augmentation de la survie du virus dans les conditions climatiques hivernales (particulièrement, la baisse de l'humidité absolue $)^{28}$ et des facteurs liés au système immunitaire ${ }^{26}$.

Selon nos travaux, si les gouvernements et les agences de santé publique veulent enrayer la transmission communautaire du SRAS-CoV-2 au cours du printemps 2021, avant la vaccination à grande échelle, des interventions non pharmacologiques strictes pourraient être nécessaires. Le Manitoba, qui a abaissé suffisamment la mobilité pour atteindre une maîtrise du SRAS-CoV-2 à l'automne, y est arrivé en imposant à toute la province un niveau de confinement parmi les plus stricts dès le 12 novembre 2020. Les mesures incluaient de limiter à 5 le nombre de personnes dans les rassemblements privés, de fermer les commerces non essentiels et les salles à manger des restaurant ${ }^{29}$ et d'appliquer plus strictement ces mesures (amendes totalisant près de 1 million de dollars imposées en date du début janvier 2021) ${ }^{30,31}$.

\section{Limites de l'étude}

Nous n'avons pas examiné les modes de mobilité granulaires au niveau des provinces, ce qui limite la capacité d'observer l'efficacité des approches régionales appliquées dans certaines provinces. Nous avons utilisé des mesures comparatives de la mobilité par rapport au niveau de janvier 2020 plutôt que par rapport à des nombres absolus, ce qui a rendu les comparaisons interprovinciales plus difficiles à faire. En outre, les rapports de Google sur la mobilité de la communauté pourraient ne pas être représentatifs de la population ontarienne dans son ensemble, et les données compilées par le Canadian Open Data Working Group n'ont pas été formellement validées. Les taux de croissance des cas de SRASCoV-2 que nous avons observés pourraient être dépendants d'une variable temporelle et sous-estimer l'influence des coefficients de variation; les tests diagnostiques des modèles ont suggéré que l'autocorrélation était faible. La température a été mesurée de manière brute pour les villes les plus 


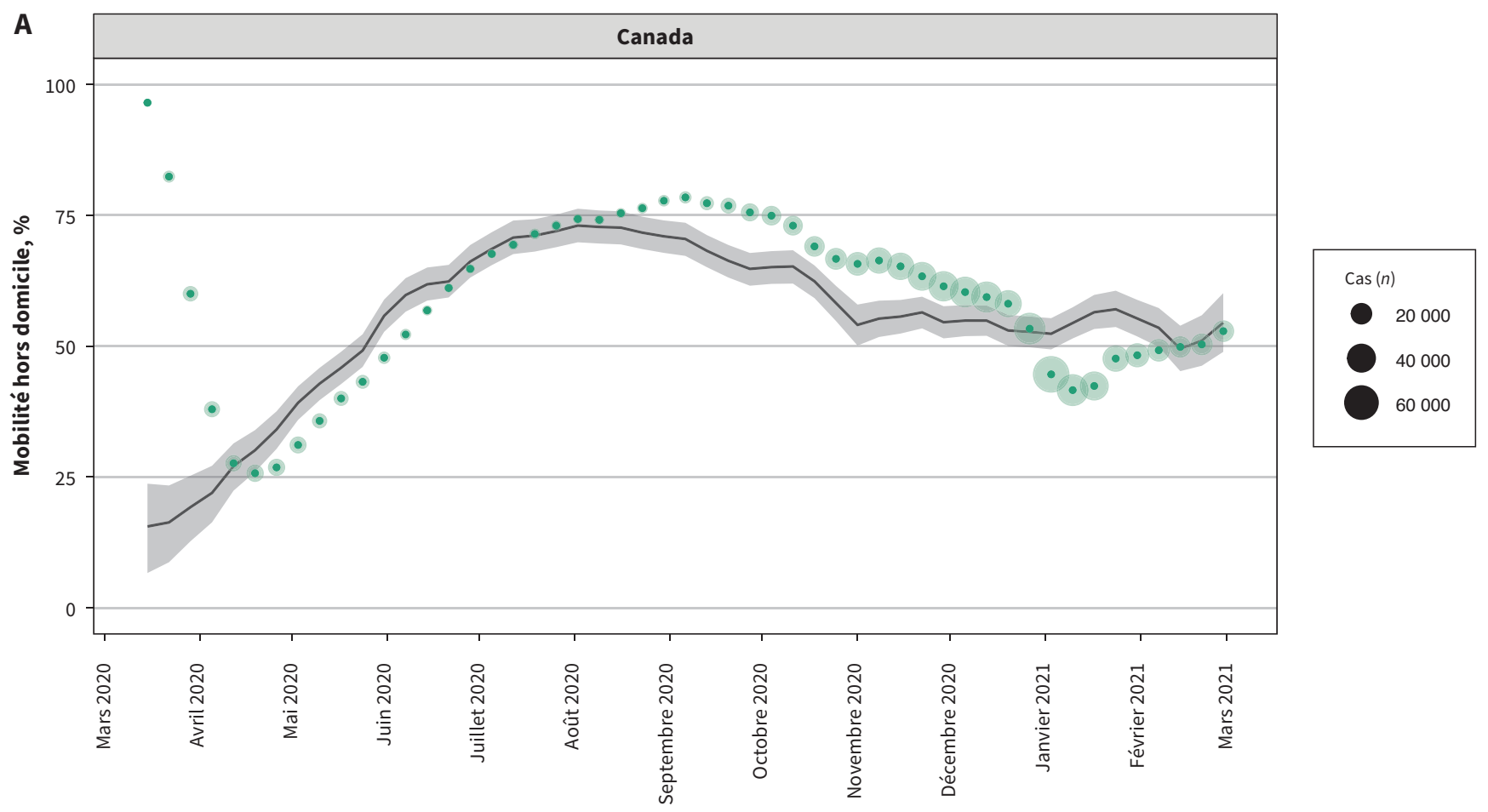

Date
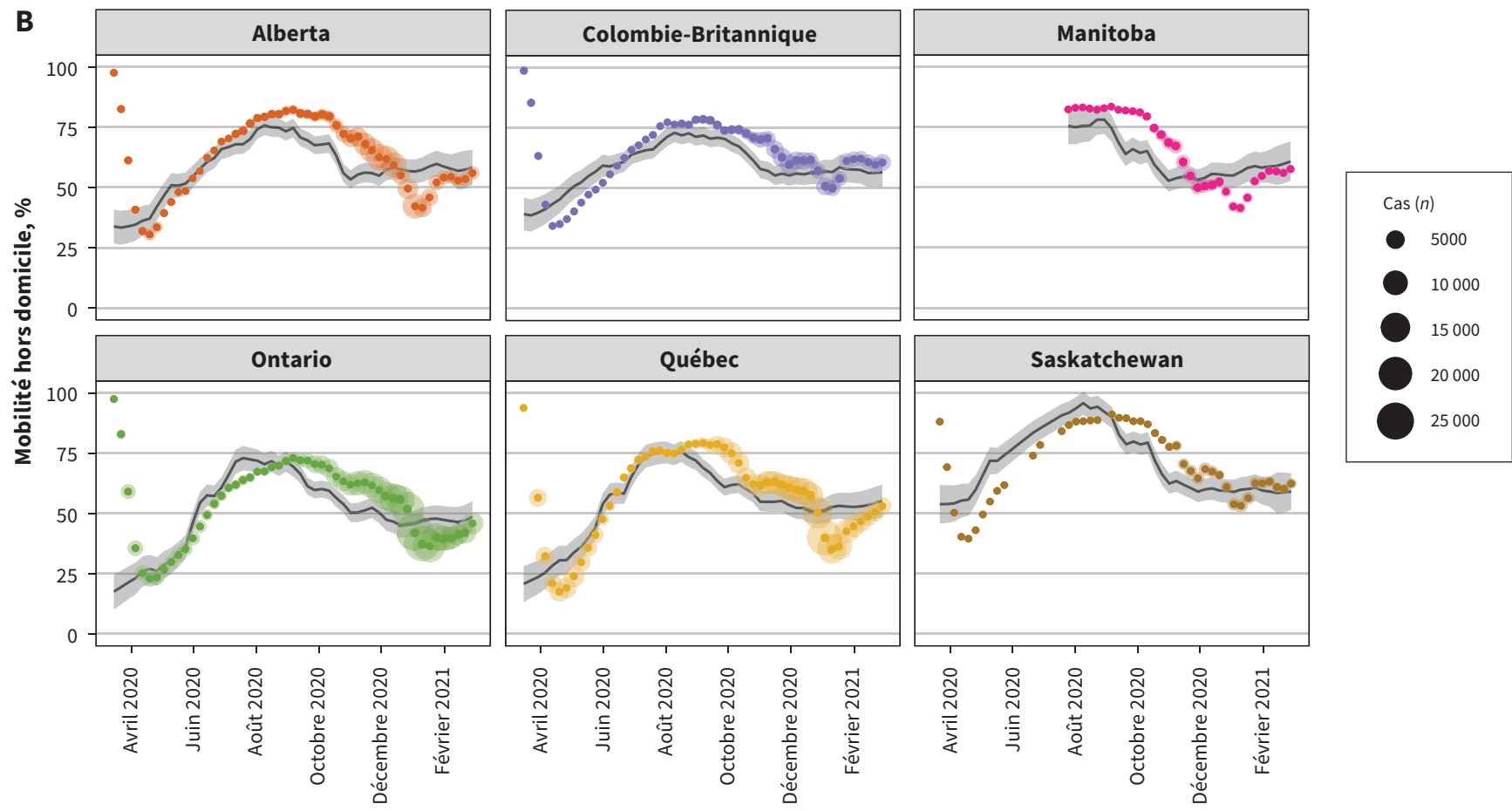

Date

Figure 3 : Variation de la moyenne mobile de la mobilité sur 3 semaines (points de couleur), seuil de mobilité estimé (ligne noire) et intervalles de confiance à $80 \%$ (zone ombrée) pour (A) le Canada et (B) 6 provinces canadiennes. La taille des cercles est proportionnelle au nombre de cas dans une semaine donnée. Remarque : Le seuil de mobilité est le niveau estimé de mobilité requis pour maîtriser la croissance des cas de coronavirus du syndrome respiratoire aigu sévère 2 (SRAS-CoV-2). Ce seuil est plus élevé l'été et plus bas dans les provinces plus populeuses, particulièrement en Ontario (médiane $50 \%$ ) et au Québec (médiane 54\%). Lorsque la mobilité est passée sous le seuil de mobilité au printemps 2020 et à l'hiver 2021, le nombre de cas hebdomadaires de SRAS-CoV-2 a diminué. À la fin novembre 2020, le Manitoba était la seule province à avoir franchi avec succès le seuil de mobilité, ce qui a entrainé des réductions de la croissance des cas de SRAS-CoV-2. Les autres provinces n'ont atteint ce seuil qu'à la fin décembre 2020 ou au début janvier 2021. 
populeuses de la province. Nous n'avons tenu compte que d'un nombre limité de variables de confusion potentielles et n'avons procédé à aucun ajustement en fonction des niveaux de vaccination contre le SRAS-CoV-2. Les vaccins ont commencé à être administrés au Canada le 14 décembre 2020, et la vaccination était encore bien en deçà des niveaux requis pour l'immunité collective en date de mars 2021. À mesure que les taux de vaccination augmenteront, ils pourraient être intégrés aux modèles de prévision du seuil de mobilité. Entretemps, la propagation rapide des variants en provenance du Royaume-Uni et de l'Afrique du Sud ${ }^{32}$ pourrait forcer une baisse du seuil de mobilité pour maîtriser la propagation de SRAS-CoV-2.

\section{Conclusion}

Cette étude montre que la mobilité est en étroite corrélation avec le taux de croissance des cas de SRAS-CoV-2 des 3 semaines subséquentes, et que des mesures strictes continueront d'être nécessaires pendant le printemps 2021 au Canada. Le seuil de mobilité et l'écart de mobilité peuvent être utilisés par les responsables de la santé publique et les gouvernements pour évaluer le degré de confinement requis pour maîtriser la propagation du SRAS-CoV-2 et guider en temps réel le déploiement et l'intensité des interventions non pharmacologiques de santé publique pour maîtriser la pandémie de COVID-19.

\section{Références}

1. Ferguson N, Laydon D, Nedjati Gilani G, et al. Report 9: Impact of nonpharmaceutical interventions (NPIs) to reduce COVID19 mortality and healthcare demand. London (UK): Imperial College London; 2020. Accessible ici : http://spiral.imperial.ac.uk/handle/10044/1/77482 (consulté le 3 mai 2020).

2. Leger's Weekly Survey - October 6, 2020. Leger 2020. Accessible ici : https:// leger360.com/surveys/legers-weekly-survey-october-6-2020/ (consulté le 16 déc. 2020).

3. Seven in ten Canadians (72\%) support four-week closure of non-essential businesses as Canada's biggest city goes back into lockdown; support in Toronto at $76 \%$. Toronto: Ipsos; 2020. Accessible ici : www.ipsos.com/ en-ca/news-polls/seven-in-ten-canadians-support-four-week-closure-of -non-essential-businesses (consulté le 16 déc. 2020).

4. Klompas M, Baker MA, Rhee C. Airborne Transmission of SARS-CoV-2: theoretical considerations and available evidence. JAMA 2020;324:441-2.

5. Kucharski A. The rules of contagion: why things spread - and why they stop. New York: Basic Books; 2020.

6. Leech JA, Nelson WC, Burnett RT, et al. It's about time: a comparison of Canadian and American time-activity patterns. J Expo Anal Environ Epidemiol 2002;12:427-32.

7. Matz CJ, Stieb DM, Davis K, et al. Effects of age, season, gender and urban-rural status on time-activity: Canadian Human Activity Pattern Survey 2 (CHAPS 2). Int J Environ Res Public Health 2014;11:2108-24.

8. Caceres N, Romero LM, Benitez FG. Exploring strengths and weaknesses of mobility inference from mobile phone data vs. travel surveys. Transportmetrica A: Transp Sci 2020;16:574-601.

9. Armstrong DA II, Lebo MJ, Do Lucas J. COVID-19 policies affect mobility behaviour? Evidence from 75 Canadian and American cities. Can Public Policy 2020;46(Suppl 2):S127-44.

10. Kishore N, Kiang MV, Engø-Monsen K, et al. Measuring mobility to monitor travel and physical distancing interventions: a common framework for mobile phone data analysis. Lancet Digit Health 2020;2:e622-8.
11. Soucy J-PR, Sturrock SL, Berry I, et al. Estimating effects of physical distancing on the COVID-19 pandemic using an urban mobility index. medRxiv 2020 May 24. doi: 10.1101/2020.04.05.20054288.

12. Badr HS, Du H, Marshall M, et al. Association between mobility patterns and COVID-19 transmission in the USA: a mathematical modelling study. Lancet Infect Dis 2020;20:1247-54.

13. Yang J, Allen A, Bailey A. What cellphone mobility data can teach us about why lockdown might not be working, and what to expect from the holidays. The Toronto Star 2020 Dec. 13. Accessible ici : www.thestar.com/ news/gta/2020/12/13/what-cellphone-mobility-data-can-teach-us-about -whos-driving-covid-infections-in-toronto-and-what-to-expect-from-the -holidays.html (consulté le 5 mars 2021).

14. Hamidi S, Ewing R, Sabouri S. Longitudinal analyses of the relationship between development density and the COVID-19 morbidity and mortality rates: Early evidence from 1,165 metropolitan counties in the United States. Health Place 2020;64:102378.

15. Berry I, Soucy J-PR, Tuite A, et al.; COVID-19 Canada Open Data Working Group. Open access epidemiologic data and an interactive dashboard to monitor the COVID-19 outbreak in Canada. CMAJ 2020;192:E420.

16. Bukhari Q, Jameel Y, Massaro JM, et al. Periodic oscillations in daily reported infections and deaths for coronavirus disease 2019. JAMA Netw Open 2020;3 e2017521.

17. COVID-19 Community Mobility Reports. Google. Accessible ici : www.google. com/covid19/mobility/ (consulté le 19 déc. 2020).

18. Li Y, Campbell H, Kulkarni D, et al.; Usher Network for COVID-19 Evidence Reviews (UNCOVER) group. The temporal association of introducing and lifting non-pharmaceutical interventions with the time-varying reproduction number (R) of SARS-CoV-2: a modelling study across 131 countries. Lancet Infect Dis 2021;21:193-202.

19. Wood SN. Generalized additive models: an introduction with R. Boca Raton (FL): Chapman \& Hall/CRC; 2006:1-391.

20. LaZerte SE, Albers S. weathercan: download and format weather data from Environment and Climate Change Canada. J Open Source Softw 2018;3:571.

21. Pedersen EJ, Miller DL, Simpson GL, et al. Hierarchical generalized additive models in ecology: an introduction with mgcv. PeerJ 2019;7:e6876.

22. Haug N, Geyrhofer L, Londei A, et al. Ranking the effectiveness of worldwide COVID-19 government interventions. Nat Hum Behav 2020;4:1303-12.

23. Brauner JM, Mindermann S, Sharma M, et al. Inferring the effectiveness of government interventions against COVID-19. Science 2021;371:eabd9338.

24. Chang S, Pierson E, Koh PW, et al. Mobility network models of COVID-19 explain inequities and inform reopening. Nature 2021;589:82-7.

25. Nouvellet $\mathrm{P}$, Bhatia $\mathrm{S}$, Cori $\mathrm{A}$, et al. Reduction in mobility and COVID-19 transmission. Nat Commun 2021;12:1090.

26. Moriyama M, Hugentobler WJ, Iwasaki A. Seasonality of respiratory viral infections. Annu Rev Virol 2020;7:83-101.

27. Fisman DN. Seasonality of infectious diseases. Annu Rev Public Health 2007;28: 127-43.

28. Shaman J, Kohn M. Absolute humidity modulates influenza survival, transmission, and seasonality. Proc Natl Acad Sci U S A 2009;106:3243-8.

29. Geary A. Manitoba's partial lockdown moved it just below COVID-19 worst-case scenario numbers. CBC News Manitoba 2020 Dec. 4. Accessible ici : www.cbc. ca/news/canada/manitoba/manitoba-covid-19-projections-1.5828626 (consulté le 22 déc. 2020).

30. MacLean C. Manitoba more than doubles fines for people who violate COVID-19 orders. CBC News Manitoba 2020 Oct. 21. Accessible ici : www.cbc.ca/news/canada/manitoba/manitoba-covid-19-enforcement -fines-increasing-1.5770947 (consulté le 11 janv. 2021).

31. New Year's Eve socializers dinged $\$ 14 \mathrm{~K}$ in fines in Winnipeg. $C B C$ News Manitoba 2021 Jan. 5. Accessible ici : www.cbc.ca/news/canada/manitoba/ new-year-eve-parties-tickets-covid-19-winnipeg-1.5861628 (consulté le 11 janv. 2021).

32. Wise J. Covid-19: new coronavirus variant is identified in UK. BMJ 2020;371: $\mathrm{m} 4857$. 
Intérêts concurrents : Kevin Brown, Nathan Stall et Peter Jüni sont affiliés à l'Ontario COVID-19 Science Advisory Table. Peter Jüni est membre non rémunéré d'un groupe qui dirige plusieurs essais cliniques subventionnés par Appili Therapeutics, AstraZeneca, Biotronik, Biosensors, Eli Lilly, St. Jude Medical et The Medicines Company, et a participé à des comités consultatifs ou agi à titre de consultant pour Amgen, Ava et Fresenius, indépendamment des travaux soumis. Aucun autre intérêt concurrent n'a été déclaré.

Cet article a été révisé par des pairs.

Affiliations : Santé publique Ontario (Brown, Buchan, Daneman); École de santé publique Dalla Lana (Brown, Soucy, Buchan, Sturrock, Berry) et Institut de gestion, d'évaluation et de politiques de santé (Stall, Jüni, Daneman), Université de Toronto; Centre de recherche appliquée en santé, Hôpital St. Michael (Jüni); Système de santé Sinai et hôpitaux du Réseau universitaire de santé; Hôpital Women's College (Stall); Département de médecine (Stall, Daneman), Université de Toronto, Toronto,
Ont.; Centre de recherches sur les communications Canada (Ghasemi); Agence de la santé publique du Canada (Gibb). Institut de recherche de l'Hôpital d'Ottawa (MacFadden), Ottawa, Ont.; Division d'infectiologie (Daneman), Institut de recherche Sunnybrook, Toronto, Ont.

Collaborateurs : Kevin Brown a conçu et modélisé le travail. Kevin Brown, Jean-Paul Soucy et Isha Berry ont acquis les données que Kevin Brown a analysées et interprétées. Kevin Brown a rédigé le manuscrit et tous les auteurs en ont révisé de façon critique le contenu intellectuel important; ils ont donné leur approbation finale pour la version destinée à être publiée et assument l'entière responsabilité de tous les aspects du travail.

Propriété intellectuelle du contenu : II s'agit d'un article en libre accès distribué conformément aux modalités de la licence Creative Commons Attribution (CC BY-NCND 4.0), qui permet l'utilisation, la diffusion et la reproduction de tout médium à la condition que la publication originale soit adé- quatement citée, que l'utilisation se fasse à des fins non commerciales (c.-à-d., recherche ou éducation) et qu'aucune modification ni adaptation n'y soit apportée. Voir : https:// creativecommons.org/licenses/by-nc-nd/4.0/ deed.fr.

Partage des données: Toutes les données utilisées pour cette étude sont du domaine public. Les données de mobilité sont disponibles ici : https://www.google.com/ covid19/mobility/. Les données sur l'incidence sont disponibles ici : https:// opencovid.ca/. Les données sont aussi disponibles sur demande.

Déclaration d'intérêts : Nathan Stall est corédacteur pour le JAMC et n'a pas participé au processus ayant mené au choix de cet article.

Accepté : Le 22 mars 2021

Correspondance : Kevin Brown, kevin.brown@utoronto.ca; Nick Daneman, nick.daneman@sunnybrook.ca 